\title{
Unilateral temporal myositis heralding polymyositis: ultrasonographic and elastographic findings. Case report.
}

\author{
Laura Damian', Carolina Botar Jid ${ }^{2}$, Liliana Rogojan ${ }^{3}$, Cristian Dinu ${ }^{4}$, Alma Maniu ${ }^{5}$, \\ Daniela Fodor ${ }^{6}$, Simona Rednic ${ }^{7}$, Siao-pin Simon ${ }^{7}$
}

${ }^{1}$ Rheumatology Department, Emergency Clinical County Hospital, ${ }^{2}$ Radiology Department, "Iuliu Hațieganu" University of Medicine and Pharmacy, ${ }^{3}$ Pathology Department, Emergency Clinical County Hospital, ${ }^{4}$ Maxillofacial Surgery Department, "Iuliu Hațieganu" University of Medicine and Pharmacy, ${ }^{5}$ ENT Department, "Iuliu Hațieganu" University of Medicine and Pharmacy, ${ }^{6}{ }^{\text {nd }}$ Internal Medicine Department, "Iuliu Haţieganu" University of Medicine and Pharmacy, ${ }^{7}$ Rheumatology Department, "Iuliu Hațieganu” University of Medicine and Pharmacy, Cluj-Napoca, Romania

\begin{abstract}
Temporal myositis is a rare inflammatory disease of the temporal muscle. We report a case of unilateral temporal myositis, in which a polymyositis was diagnosed two years thereafter. Although focal myositis may rarely herald polymyositis, isolated temporal myositis preceding inflammatory myopathies has not been described, to our knowledge. In the setting of a temporal pain and swelling, ultrasonography may help in diagnosis, biopsy guidance, disease extension, and progression assessment. Further studies are necessary to establish the role of elastography in differentiating between muscle inflammation and hypertrophy.
\end{abstract}

Keywords: temporal myositis, polymyositis, ultrasonography, elastography.

\section{Introduction}

Temporal myositis (TM) is a rare inflammatory disease of the temporal muscle evolving with local swelling and tenderness [1]. Ultrasonography is a reliable method in the assessment of temporal muscles pathology [2-5]. Moreover, temporal artery Doppler ultrasound is currently used to guide and even to replace biopsy in giant cell arteritis [6].

There are few reports of masticator muscle ultrasound in systemic diseases. Localized or focal myositis may rarely herald a polymyositis $[7,8]$.

Received 12.09.2015 Accepted 31.10.2015

Med Ultrason

2016, Vol. 18, No 1, 123-126

Corresponding author: Carolina Botar Jid

Radiology Departament,

Emergency Clinical County Hospital,

3-5 Clinicilor street

400006, Cluj-Napoca, Romania

E-mail: inabotar@yahoo.com

\section{Case report}

A 22-year old female patient, with no significant previous history, was sent by the general practitioner in September 2008 for a painful swelling of the right temporal region. The general examination did not reveal any other abnormality, oral or systemic. The peripheral pulses were equal, no carotidian bruit or other signs or symptoms suggestive of Takayasu's disease or polymyositis were observed. The carotidian Doppler ultrasound was also normal. The laboratory analyses revealed a mildly increased erythrocytes sedimentation rate (ESR $20 \mathrm{~mm} / \mathrm{h}$ ), leukocytosis with left shift (WBC 11000, with 78\% neutrophils) and a slight creatine phosphokinase (CPK) elevation (198 IU, normal values $<164$ ). The temporomandibular joint was clinically and radiologically normal. A stomatological examination found no abnormalities, nor occlusive dysfunction. The biopsy revealed temporal myositis, without ossification. She was started on NSAIDS, with some improvement. Still, because of the aesthetic discomfort due to the persistent swelling, methotrexate was attempted and stopped due to intolerance. 
After two years she presented again with temporal pain, along with proximal muscular asthenia. A workup for polymyositis revealed elevated CPK (350 IU, normal values $<164$ ) and electromyography was consistent with myopathic findings. The laboratory revealed mild leukocytosis (WBC 10500), a C-reactive protein of $2.1 \mathrm{mg} /$ $\mathrm{dl}$ (normal values $<0.6$ ), an antinuclear antibody titer of $1 / 160$, with spotted pattern, no complement consumption and negative ANCA, anticardiolipin IgG antibodies, HBV, HCV, HIV and Trichinella serology. The immunoblot for extractable nuclear antigens (ENA) was negative, as well as the one for myositis (Ro, La, U1RNP, Jo-1, Ku, SRP, Pm/Scl 75, Pm/Scl-100). Again, a Takayasu disease workup (clinical, Doppler ultrasound and magnetic resonace angiography) was non-contributive. Ultrasound examination (performed using a Hitachi EUB 8500 machine equipped with multifrequency linear transducer 6.5-13 MHz, with software for bidimensional and Doppler ultrasound, and strain elastography respectively) revealed an enlarged right temporal muscle (fig 1) compared to the contralateral side. No important alteration of the regular comb-like pattern of the muscular fibers was noted, apart from a discrete deletion of fibrillar muscular appearance. She was started on corticotherapy, hydroxychloroquine and azathioprine (switched to cyclosporine due to intolerance), with control of the systemic disease. Temporal muscle strain elastography repeatedly performed during therapy revealed no stiffness, rather suggesting the lack of progression to fibrosis (fig 2).

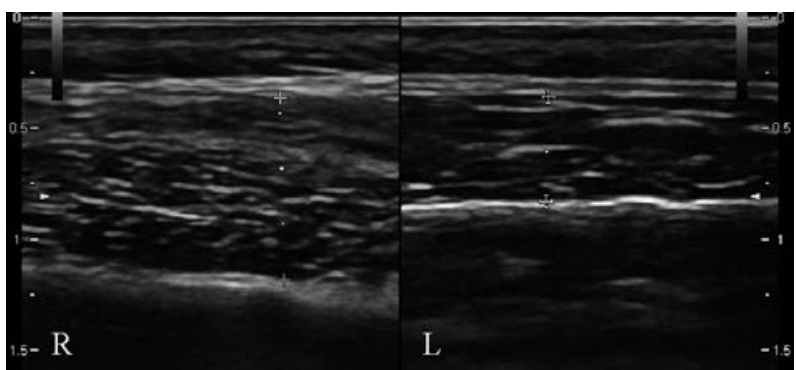

Fig 1. Right temporal muscle 2D US: enlarged right temporal muscle (R) comparing with the left side (L), with no important alteration of muscular structure

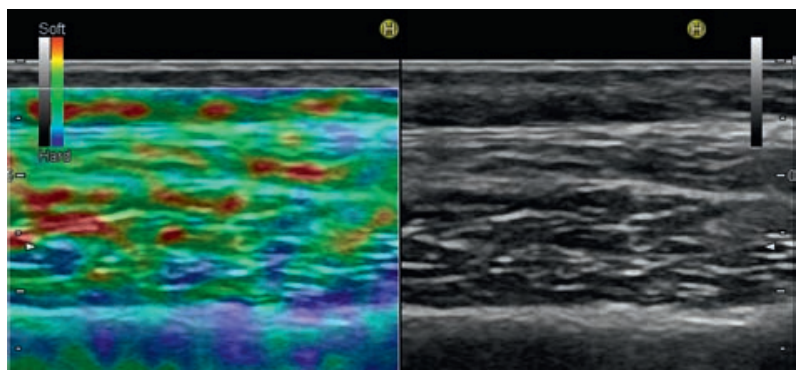

Fig 2. Right temporal muscle strain elastography: moderate elasticity with striated appearance

\section{Discussion}

TM is a rare finding in the masticatory muscles [1]. Clinical symptomatology may involve, besides pain, a rapidly enlarging swelling and asymmetry of the involved area; trismus and fever may be present. In localized TM blood tests may show, as in our case, signs of mild inflammation [9]. Histology reveals inflammation and necrosis [9].

Most often TM is due to posttraumatic myositis ossificans (MO), after dental therapy or other types of head and neck surgery [10]. Bilateral MO of the masseter muscles followed chemoradiotherapy and critical illness neuropathy [11]. TM after surgery employing a titanium plate and screws covered by muscles was possibly due to autoimmune syndrome induced by adjuvants (ASIA) syndrome $[10,12]$. Masticatory myositis may be the presenting sign of trichinosis or cysticercosis [5] and may rarely complicate otitis media [13].

Masticatory muscles are subclinically involved in inflammatory myopathies [14]. Localized nodular myositis may seldom be the first manifestation of polymyositis $[7,8]$. However, TM heralding polymyositis has not been previously reported, to our knowledge. Localized myositis may be a symptom of vasculitis $[15,16]$. In our case, the temporal symptomatology leads to an extensive search for Takayasu arteritis, which was excluded.

The differential diagnosis of temporal pain includes, besides Takayasu or Horton arteritis, temporomandibular dysfunction, temporomandibular arthritis and various headache types. Myofascial pain may cause masticatory myalgia, when a trigger point can be identified. TEM should be differentiated from muscle hypertrophy ordystrophy, eosinophilic fasciitis, lipomatosis, oedema, haemmorhage, abscess, cavernous hemangioma, infiltrative leukemia, lymphoma, liposarcoma, rhabdomyoma, chondromas and osteochondroma [17].

In evolution, focal TM may regress spontaneously [9]. Nevertheless, evolution to a systemic disease should be considered. The therapy consists of NSAIDs and second-line drugs, if systemic signs are present. In our case the immunosuppressive treatment controlled the systemic as well as the local disease.

The cause of right temporal muscle hypertrophy in our case is, however, not clear. The right temporal muscle painful enlargement was noted from the disease onset, as in another TM case [1]. Early-stage polymyositis may result in muscle hypertrophy [18]. Unilateral reactive painful hypertrophy of the temporal muscle has been rarely reported, associated with bruxism, malocclusion, or temporomandibular joint 
disease [17]. Our patient was exercising the masticatory muscles in order to compensate for the discrete facial asymmetry due to TM.

A further temporal biopsy was refused by the patient. Ultrasonography as a non-invasive procedure was employed instead, as a reliable method of measurement of masseter and temporalis muscle thickness [2]. U1trasonography was reportedly useful in the diagnosis of temporal cysticercosis [5] and in the assessment of early recurrent MO [4]. The thickness of masticatory muscles significantly increases during voluntary dental clenching [3]. This should be taken into account, as well as standardizing the transducer position, when repeatedly measuring during follow-up [3].

Sonographic elastography, a relatively new technique allowing the investigation of tissue elasticity, was employed in polymyositis [19]. The method has been reproducibly used in masseter muscles, and a potential relationship may exist between elasticity and edematous change in the masseter muscle [20]. The elastographic evaluation of the propagation velocity of the shear waves using the acoustic radiation force impulse method (ARFI) masseter muscles allows quantitative measurements of normal and post-irradiation masseter elasticity [21]. The method has not been reportedly used, to our knowledge, in temporal myositis and it is still unclear whether it could make a difference between muscle inflammation and hypertrophy. However, as the hardness may increase in the presence of edema, as the muscle is covered with a thick fascia, an increase in intramuscular pressure accompanied by an increase in the water content may increase hardness [20]. In our patient, strain elastography revealed no alteration in elasticity in the right temporal muscle, and, more important, no stiffness increase in this region. This elastographic aspect suggests no evolution to fibrosis [19].

In conclusion, TM should be included in the differential diagnosis of jaw pain and motion limitations. TM heralding a polymyositis has not been reported before, to the best of our knowledge. Although frequently isolated, TM may warrant a search for signs of systemic disease. In the setting of a temporal pain and swelling, ultrasonography may help in the diagnosis, in assessing the extension of the lesion and in guiding muscle biopsy. It also contributes to the exclusion of other etiologies, such as vasculitis, tumours, infections and possibly in order to monitor disease progression. Elastography could be also a useful tool in the management of myositis, but further studies are awaited.
Aknowledgements: The authors want to thank Assoc. Prof. Dr. Adriana Albu for the carotidian Doppler examination and to Dr. Liliana Bene for the immunological analyses.

\section{References}

1. Naumann M, Toyka KV, Goebel HH, Hoffmann E, Reichmann H. Focal myositis of the temporal muscle. Muscle Nerve 1993; 16: 1374-1376.

2. Serra MD, Duarte Gaviao MB, dos Santos Uchoa MN. The use of ultrasound in the investigation of the muscles of mastication. Ultrasound Med Biol 2008; 34: 1875-1884.

3. Mangilli LD, Sassi FC, Sernik RA, Tanaka C, Andrade CR. Electromyographic and ultrasonographic characterization of masticatory function in individuals with normal occlusion. J Soc Bras Fonoaudiol 2012; 24: 211-217.

4. Okayama A, Futani H, Kyo F, Maruo S, Koezuka A, Kinoshita G. Usefulness of ultrasonography for early recurrent myositis ossificans. J Orthop Sci 2003; 8: 239-242.

5. Rastogi S, Arora P, Devi P, Wazir SS, Kapoor S. Importance of ultrasonography and magnetic resonance imaging in diagnosis of cysticercosis of temporalis muscle mimicking temporal space infection. Contemp Clin Dent 2013; 4: 504-508.

6. Diamantopoulos AP, Haugeberg G, Hetland H, Soldal DM, Bie R, Myklebust G. Diagnostic value of color Doppler ultrasonography of temporal arteries and large vessels in giant cell arteritis: a consecutive case series. Arthritis Case Res (Hoboken) 2014; 66: 113-119.

7. Brown P, Doyle DV, Evans MD. Localized nodular myositis as the first manifestation of polymyositis (letter). Br J Rheumatol 1989; 28: 84.

8. Flaisler F, Blin D, Asencio G, Lopez FM, Combe B. Focal myositis: a localized form of polymyositis? J Rheumatol 1993; 20: 1414-1416.

9. Kawasaki T, Keisuke O, Kuninori O: A patient with focal myositis of the temporal muscle. Oto-Rhino-Laryngology Tokyo 2006; 49: 230-233.

10. Ueda K, Tajima S, Shirakabe M, Ohba S, Ohmiya Y, Byun $\mathrm{S}$. A case of bilateral temporal muscle myositis accompanied by rare facial cleft. Eur J Plast Surg 1999; 22 : 132134.

11. Kruse AL, Dannemann C, Gratz KW. Bilateral myositis ossificans of the masseter muscle after chemoradiotherapy and critical illness neuropathy- report of a rare entity and review of the literature. Head Neck Oncol 2009: 1: 30.

12. Shoenfeld Y, Agmon-Levin N. 'ASIA'- autoimmune/ autoinflammatory syndrome induced by adjuvants. J Autoimmun 2011; 36: 4-8.

13. Kuczkowski I, Narozny W, Stankiewicz C, Mikasewski B, Izycka-Swieszewska E. Zygomatic abscess with temporal myositis- a rare extracranial complication of acute otitis media. Int J Pediatr Otorhinolaryngol 2005; 69: 555-559. 
14. Marton K, Hermann P, Danko K, Fejerdy P, Madlena M, Nagy G. Evaluation of oral manifestations and masticatory force in patients with polymyositis and dermatomyositis. J Oral Pathol Med 2005; 34: 164-169.

15. Allen I, Mullally B, Mawhinney H, Sawhney B, McKee P. The nodular form of polymyositis- a possible manifestation of vasculitis. J Pathol 1980; 131: 183-191.

16. Yazici H, Tuzuner N, Tuzun Y, Yurdakul S. Localized myositis in Behcet's disease. Arthritis Rheum 1981; 24: 636.

17. Katsetos CD, Bianchi MA, Jaffery F, Koutzaki S, Zarella M, Slater R. Painful unilateral temporalis muscle enlargement: reactive masticatory muscle hypertrophy. Head Neck Pathol 2014; 8: 187-193.
18. Cherin P, Herson S. Myopathies inflammatoires. In: Kahn MF, editor. Maladies et syndromes systemiques. 4the Ed, Paris, Medecine-Sciences Flammarion; 2000 : 597-622.

19. Botar-Jid C, Damian L, Dudea SM, Vasilescu D, Rednic S, Badea $\mathrm{R}$. The contribution of ultrasonography and sonoelastography in the assessment of myositis. Med Ultrason 2010; 12: 120-126.

20. Gotoh A, Ariji Y, Hasegawa T, et al. Sonographic elastography for assesing changes in masseter muscle elasticity after low-level static contraction. Oral Radiol 2013; 29: 140-145.

21. Badea I, Tamas-Szora A, Chiorean I, Fildan F, Ciulea E, Badea M. Quantitative assessment of the masseter muscle's elasticity using Acoustic Radiation Force Impulse. Med U1trason 2014; 16: 89-94. 